\title{
Important notes for clinical trials funded or sponsored by WHO
}

Citation: World Health Organization. Important notes for clinical trials funded or sponsored by WHO. East Mediterr Health J. 2018;24(5):489. https:// doi.org/10.26719/2018.24.5.500

Copyright @ C World Health Organization (WHO) 2018. Some rights reserved. This work is available under the CC BY-NC-SA 3.0 IGO license (https:// creativecommons.org/licenses/by-nc-sa/3.o/igo).

\section{Registration with clinical trial registry}

Before the clinical trial is initiated (at any Phase), its details are to be registered in a publically available, free to access, searchable clinical trial registry complying with WHO's international agreed standards (see www.who.int/ ictrp). The clinical trial registry entry is to be made before the first subject receives the first medical intervention in the trial. It is preferable that a registry is chosen with a functional results section.

\section{Publication of results}

Without prejudice to the confidentiality of proprietary information, clinical trial results are to be reported as set forth below. In the case of results that may be capable of industrial or commercial exploitation, confidentiality shall be maintained for a period of twelve months in order to enable patent rights to be safeguarded or to allow alternative forms of legal protection to be explored. Unless it is mutually agreed that confidentiality beyond a period of twelve months is necessary and consistent with the public interest, the parties shall not be bound by any obligation to keep the results confidential.

1. The clinical trial results (main findings) are to be submitted for publication in a peer reviewed journal within 12 months of study completion, and are to be published through an open access mechanism in accordance with WHO's open access policy within 24 months of study completion; and

2. Summary results of clinical trials are to be posted on the results section of the primary clinical trial registry within 12 months from primary study completion. Where a registry is used without a results database available, the results is to be posted on a free-to- access, publicly available, searchable institutional website, such as the WHO website.

The clinical trial protocol is to be made publicly available no later than the time at which the summary results are posted on the results section of the clinical trial registry. This includes amendments approved by ethics committees/institutional review boards. The protocol and amendments are to be either uploaded in electronic document formats such as PDFs, or provided as a link to electronic document formats. Study completion means the date on which the final participant in the clinical trial was examined or received an intervention for the purposes of final collection of data for the primary outcome, whether the study concluded according to the pre-specified protocol or was terminated earlier.

Where negative or inconclusive results are not expected for publication in a peer review journal, disclosure requirements will be met by point 2 above, and a final study report including all protocol specified analyses is to be developed and retained by the WHO responsible staff member for WHO sponsored clinical trials and the Principal investigator for WHO funded clinical trials.

The Trial ID or registry identifier code/number is to be included in all publications of clinical trials, and provided as a part of the abstract to PubMed and other bibliographic search databases for easy linking of trial related publications with clinical trial registry site records. This is essential for linking journal publications with registry records.

Compliance (or failure to comply) with the above mentioned requirements within the above mentioned deadlines or any extensions thereof agreed by WHO in writing, will be monitored by WHO and the details thereof may publicly be disclosed on the WHO website. 